\title{
Group size and modularity interact to shape the spread of infection and information through animal societies
}

\author{
Julian C. Evans ${ }^{1} \cdot$ David J. Hodgson ${ }^{2} \cdot$ Neeltje J. Boogert ${ }^{2} \cdot$ Matthew J. Silk ${ }^{2,3}$
}

Received: 1 May 2021 / Revised: 8 November 2021 / Accepted: 10 November 2021 / Published online: 27 November 2021

() The Author(s), under exclusive licence to Springer-Verlag GmbH Germany, part of Springer Nature 2021

\begin{abstract}
Social interactions between animals can provide many benefits, including the ability to gain useful environmental information through social learning. However, these social contacts can also facilitate the transmission of infectious diseases through a population. Animals engaging in social interactions therefore face a trade-off between the potential informational benefits and the risk of acquiring disease. Theoretical models have suggested that modular social networks, associated with the formation of groups or sub-groups, can slow spread of infection by trapping it within particular groups. However, these social structures will not necessarily impact the spread of information in the same way if its transmission follows a "complex contagion", e.g. through individuals disproportionally copying the majority (conformist learning). Here we use simulation models to demonstrate that modular networks can promote the spread of information relative to the spread of infection, but only when the network is fragmented and group sizes are small. We show that the difference in transmission between information and disease is maximised for more well-connected social networks when the likelihood of transmission is intermediate. Our results have important implications for understanding the selective pressures operating on the social structure of animal societies, revealing that highly fragmented networks such as those formed in fission-fusion social groups and multilevel societies can be effective in modulating the infection-information trade-off for individuals within them.
\end{abstract}

Keywords Social network $\cdot$ Infectious disease $\cdot$ Conformist learning $\cdot$ Social learning rule $\cdot$ Transmission

\section{Introduction}

Most animals engage in some form of social interaction (Krause et al. 2002; Frank 2007; Clutton-Brock 2016). These interactions frequently provide opportunity for gaining social information (Danchin et al. 2004; Laland 2004; McGregor 2005; Allen et al. 2013; Aplin et al. 2015), whether it be the location of new food sources (Aplin et al.

Communicated by: R. Rosengaus de Nurko

Julian C. Evans

jevansbio@gmail.com

$\triangle$ Matthew J. Silk

matthewsilk@outlook.com

1 Deparment of Evolutionary Biology and Environmental Studies, University of Zurich, Zurich, Switzerland

2 Centre for Ecology and Conservation, University of Exeter Penryn Campus, Penryn, UK

3 National Institute of Mathematical and Biological Synthesis (NIMBioS), University of Tennessee, Knoxville, TN, USA
2012; Kendal et al. 2015; Berdahl et al. 2018; Nöbel et al. 2018) or predation risk (Beauchamp et al. 2012; Crane and Ferrari 2013; Frechette et al. 2014). However, interacting with others can also be costly, with one of the primary costs being the risk of infection with pathogens or parasites (Daszak et al. 2000; Stattner and Vidot 2011). Balancing risk of infection with the potential to gain useful information can thus be one of the key trade-offs animals face when engaging in social interactions (Evans et al. 2020; Romano et al. 2020). Although it is possible to obtain information or become infected indirectly via interactions with the physical environment (e.g. scent marking: Gosling and Roberts 2001; disease reservoirs: Adelman et al. 2015), many studies have shown that the spread of both infectious disease and information through a population will be strongly influenced by social interaction patterns (Aplin et al. 2012; White et al. 2017; Hoyt et al. 2018). While the transmission of infection and information may depend on different types of interactions, there is likely to be overlap depending on how the pathogen spreads between hosts and how individuals learn from each other (Evans et al. 2020). 
To better understand this potential trade-off between information and disease, it is necessary to quantify how different social structures can promote access to useful information while minimising risk of infection (Evans et al. 2020; Firth 2020; Romano et al. 2020). Social structures can help maximise the benefits of sociality for their members by promoting the persistence of social units and so increasing their fitness indirectly (Ilany and Akcay 2016; Kramer and Meunier 2019). As such, selection can act on behaviours because of their influence on emergent social structures in addition to their direct impact on individual fitness. Consequently, investigating which aspects of sociality slow down the spread of disease while promoting information transmission therefore represents an important contribution to our understanding of social evolution.

Animal social systems vary widely among species (Krause et al. 2002). Social networks offer a useful toolkit to capture and quantify this diversity (Krause et al. 2007; Wey et al. 2008; Pinter-Wollman et al. 2014), and can help distinguish species that, for example, live in multi-level societies (e.g. reticulated giraffes Giraffa camelopardalis: VanderWaal et al. 2014; sperm whales Physeter macrocephalus: Cantor et al. 2015; vulturine guineafowl Acryllium vulturinum: Papageorgiou et al. 2019), stable social groups (e.g. meerkats Suricata suricatta: Drewe et al. 2009; European badgers Meles meles: Weber et al. 2013), fission-fusion groups (e.g. Bechstein's bats Myotis bechsteinii: Kerth and König 1999; many bird species: Silk et al. 2014; reviewed in general: Couzin and Laidre 2009), or whose interactions and associations are more constrained by other factors such as shared space or resource use (Davis et al. 2015; Spiegel et al. 2016; Evans and Morand-Ferron 2019). Social network structure plays a critical role in governing both pathogen and information transmission (Aplin et al. 2012; Godfrey 2013; Webster et al. 2013; Silk et al. 2017; Evans et al. 2020). For example, considerable variability in the connectedness of individuals can drive a superspreader effect in infectious diseases, causing more explosive outbreaks (Lloyd-Smith et al. 2005). Global properties of the network such as the number (or density) of connections and how these are distributed are also important. The efficiency of information transfer is maximised when social networks show intermediate levels of subdivision into different modules or communities (Romano et al. 2018). Many animal social networks possess such modular structures (Wey et al. 2008), especially species that form relatively stable social groups (e.g. meerkats: Drewe et al. 2009; badgers: Weber et al. 2013). Consequently, both community structure and how inter-connected these communities are - their modularity — are likely to be important in governing any differences between information and disease transmission in animal societies.

There are often important differences in how infection and information spread through a network. Infection is generally considered a simple contagion (Moore and Newman 2000); the likelihood of becoming infected will depend predominantly on the number of relevant contacts with infected individuals, and the duration of these contacts (Godfrey et al. 2009). In contrast, information can often be considered a complex contagion (Macy 1991; Centola 2010; Firth 2020); individuals decide on whether to act on the information acquired and so may use different social learning strategies that change how information spreads (Laland 2004; Kendal et al. 2018). For example, they might accept information only from certain individuals (e.g. Kavaliers et al. 2005; van de Waal et al. 2010), or be much more likely to use it if a set proportion of their contacts behave in that way (Danchin et al. 2018). The latter can result in conformist social learning, which has been demonstrated in taxonomically diverse animal societies (e.g. vervet monkeys Chlorocebus aethiops: van de Waal et al. 2013; great tits Parus major: Aplin et al. 2015; fruit flies Drosophila melanogaster: Danchin et al. 2018).

Because of the differences in how infection and information spread, modularity shapes their transmission in different ways (Nematzadeh et al. 2014; Sah et al. 2017; Evans et al. 2020). While modular networks can promote the spread of infection within particular groups, they tend to trap infection within these groups, slowing down disease spread through the population as a whole (Sah et al. 2017). The impact of modular structure on disease outbreaks is greatest when the modularity of these subdivisions is high (i.e. different groups have relatively few contacts between them; Salathé and Jones 2010; Sah et al. 2017), especially when transmissibility is low so that infection is unlikely through occasional contacts (Griffin and Nunn 2012; Sah et al. 2017; Rozins et al. 2018). In contrast, when information transmission is a complex contagion where individuals disproportionally copy the majority of their group members (Aplin et al. 2015), modular networks can promote the global spread of information, especially at intermediate modularities (i.e. when contacts between groups or modules are infrequent but not rare), due to strong social reinforcement within groups (Nematzadeh et al. 2014). As a result, modular networks may provide one route to promote the spread of information through a population disproportionately to the spread of infection (Evans et al. 2020). However, there are many other aspects of the structure of modular networks that might also shape this pattern and have been less well studied.

Social group (or sub-group) size, which is highly variable across and within animal societies, represents one such trait that could also impact how network structure shapes transmission dynamics (Côté and Poulinb 1995; Nunn et al. 2015). Sah et al. (2017) showed that modular networks caused the greatest reduction in epidemic spread when networks were composed of many smaller (sub-) groups, i.e. network fragmentation was high (Godfrey 
et al. 2009; Sah et al. 2017). This suggests that the formation of more fragmented networks consisting of smaller rather than larger (sub-)groups is more effective in reducing infectious disease transmission. However, different levels of network fragmentation may also change the optimum modularity for information transmission, which depends on high clustering of connections within groups to promote the spread of information in the first place (Nematzadeh et al. 2014). For example, small modules or sub-groups that contain many friends of friends may help promote the spread of information while limiting infectious disease transmission if between-group contacts are rare. This could be especially important as the impact of modularity on infection transmission is non-linear, with modular networks having no substantial effect on how disease spreads when modularity is below a certain value (Sah et al. 2017). Thus, if the optimal level of separation between groups to promote information spread is too far below the level of separation at which a modular network structure begins to protect effectively against the spread of infectious disease, then the benefits are lost.

We used simulation models to examine how social group size (network fragmentation) interacts with modularity (the strength of the subdivision of a network into groups) to determine differences in the speed of social information spread and infectious disease transmission. We generated unweighted (binary) networks of a fixed size with different edge density (the social connectedness of individuals), group sizes (size of social groups within the network), and modularity (strength of the division between these social groups) to examine how these properties affected the transmission rates of information and disease, at varying levels of transmissibility. We treated the spread of information using a conformist model in which the relationship between the likelihood of acquiring/using information and proportion of informed contacts was sigmoidal, while infection risk was fixed for each contact with an infected individual. We compared the rates of spread of infection and information through social networks that varied in both their modularity and the size of groups making up the modular structure of the network, for different values of social connectedness and transmissibility (how likely a disease or a piece of information is to be transmitted). We expected that modular networks would promote the transmission of information relative to the spread of infection, especially when transmissibility was low. However, because of previous research suggesting a role of network fragmentation alongside modularity on transmission dynamics (Sah et al. 2017), we anticipated that the extent of this benefit would also be shaped by the number and size of social groups, although we had no a priori expectation for the direction of this effect.

\section{Methods}

\section{Simulation summary}

Each simulation consisted of two main steps (Fig. 1). First, we generated random networks which were used to select parameters controlling disease and information spread. We then altered these random networks to create modular networks of varying levels of fragmentation, in which we compared relative transmission speeds (for infection and information spreading independently).

We generated a random network of 200 nodes (i.e. individuals) with a particular edge density (proportion of potential pairs of individuals in the network who were connected; Table 1). We then simulated the spread of a disease through this network, starting from a randomly selected individual, using a susceptible-infected (SI) model with the transmissibility of that disease in that network derived from an adjusted, per time interval $R_{0}$ (infectiousness of the disease; Table 1). Values of $R_{0}$ were chosen to simulate a broad range of pathogens with transmissibilities that have previously been shown to generate disease dynamics that can be influenced by modular network structure (Sah et al. 2017; Rozins et al. 2018). This disease simulation was carried out in the random network 50 times, each time starting with a different randomly selected individual (50 simulations sufficed for our results not to be influenced by the randomly selected starting individual; Supplementary Fig. 1). For each of these simulations, we recorded the number of timesteps it took a disease to infect $75 \%$ of the individuals in that network, as it may not be possible for $100 \%$ of individuals to be infected in some of our networks. We used the distribution of timesteps taken to reach this $75 \%$ threshold in the 50 disease simulations to determine the value of a scaling parameter $\gamma$, which modified the speed of information spread, so that information spread at a similar speed to disease in the random network. Matching the speeds of the two transmission processes in the random networks allowed us to isolate the effects of network fragmentation and modularity when simulating spreads in modular networks. We simulated information spread using this scaling parameter through the random network 50 times, starting with a random node, and recorded the average time taken for $75 \%$ of individuals to be informed.

We then rewired the edges of the random network to produce new modular networks with the same edge density but a particular network modularity (strength of division of a network into modules; Table 1), with individuals subdivided into social groups of a particular size. This led to a total of 18 modular networks being derived from each random network, one for every combination of modularity and group size (see Table 1 for values used). We simulated the spread of disease and information through each 
Fig. 1 Summary of simulations for a single parameter set

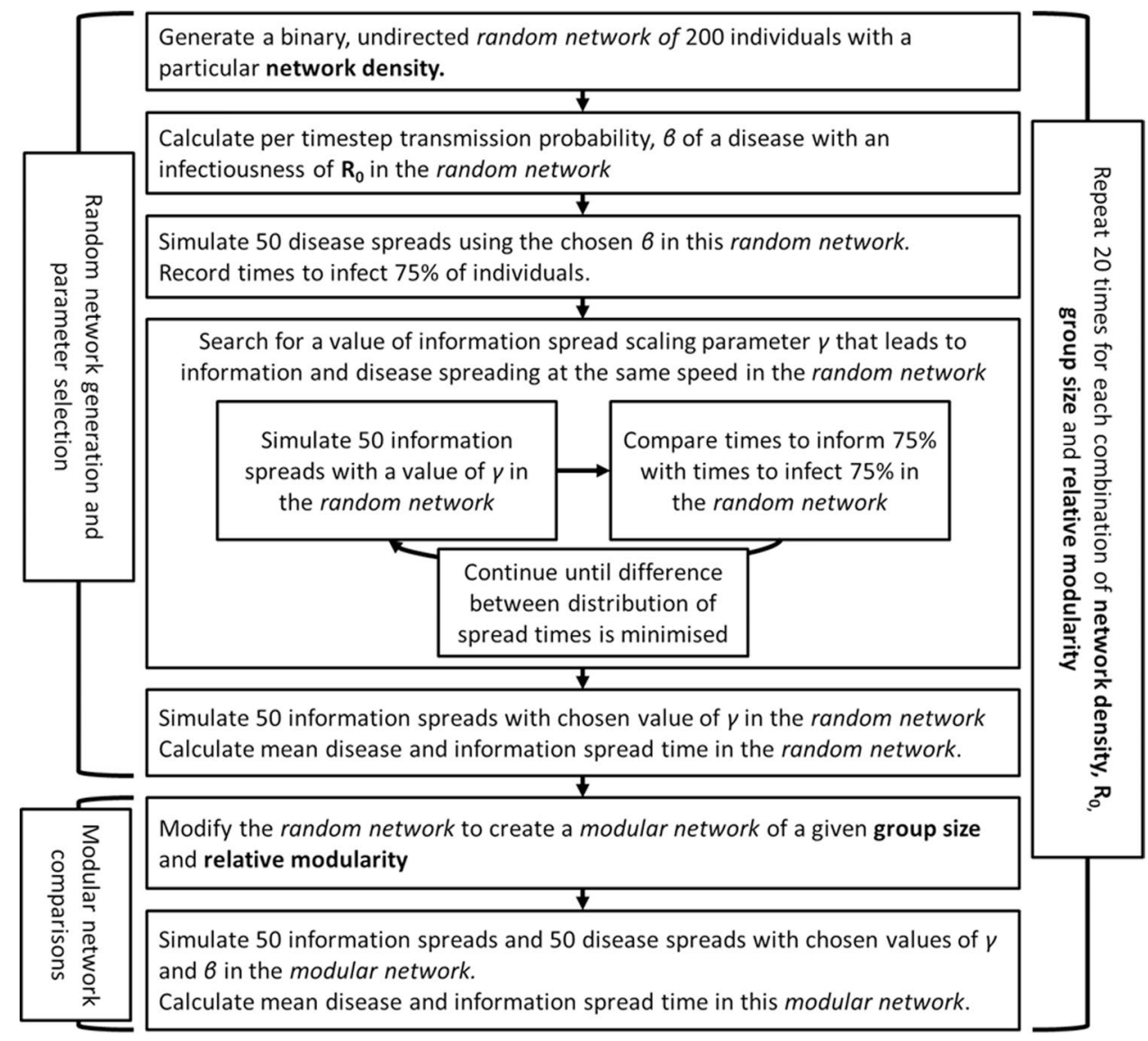

Table 1 Description of the four parameters used in the simulations. Parameters are summarised in bold, followed by more detailed explanation. Number of parameter values and values also shown

\begin{tabular}{|c|c|c|c|}
\hline Parameter & Description & No. of values & Values \\
\hline Network density & $\begin{array}{l}\text { Proportion of potential connections existing in the network } \\
\text { Higher densities result in more edges in the initial random network and in derived } \\
\text { modular networks. A high network density represents a population that is more } \\
\text { closely interconnected, where individuals interact with a greater proportion of } \\
\text { the population on average }\end{array}$ & 9 & $\begin{array}{l}0.02,0.03,0.04,0.05 \\
0.06,0.07,0.08 \\
0.09,0.1\end{array}$ \\
\hline$R_{0}$ & $\begin{array}{l}\text { Basic reproduction number (per timestep), infectiousness of disease } \\
\text { Used to derive values of } \beta \text { (transmission probability in a particular random net- } \\
\text { work) and } \gamma \text { (scaling parameter to ensure information spreads at the same rate as } \\
\text { disease in that random network). Values cover pathogens ranging from weakly } \\
\text { (e.g. some influenza strains) to moderately transmissible (e.g. SARS-CoV 2) }\end{array}$ & 5 & $1.1,1.25,1.5,2,3$ \\
\hline Group size & $\begin{array}{l}\text { Social group size/Network fragmentation } \\
\text { Controls the size of social groups in the modular networks derived from a random } \\
\text { network. Chosen values represent a range of biologically realistic group sizes. } \\
\text { Networks with smaller groups were more fragmented }\end{array}$ & 6 & $5,8,10,20,25,40$ \\
\hline Network modularity & $\begin{array}{l}\text { Strength of division of a network into modules } \\
\text { Level of separation between groups. Larger values result in less intergroup edges } \\
\text { in the modular networks derived from a random network. i.e. a population more } \\
\text { strongly divided into groups, with fewer between-group social connections }\end{array}$ & 3 & $0.4,0.6,0.8$ \\
\hline
\end{tabular}

of these new modular networks 50 times, recording the average time each process took to infect or inform $75 \%$ of nodes in the rewired, modular, networks. We then compared the difference in mean time taken for information and infection to reach the $75 \%$ threshold in that particular modular network. We also compared the transmission rates through a modular network to those through the random network from which it was derived, to establish the relative influence of modularity and group sizes on the transmission speeds of information and disease. 
The nine values of network density, five values of transmissibility, six values of group size (network fragmentation), and three values of network modularity tested led to a total of 810 parameter combinations (Table 1). Each parameter combination was repeated 20 times. We conducted all 16,200 independent simulation runs (each containing multiple simulated spreads of infection and information) in $\mathrm{R}$ 4.0.2 ( $\mathrm{R}$ Development Core Team 2020). $\mathrm{R}$ code is provided in the Supplementary Materials. See Supplementary Video 1 for a visualisation of an example simulation.

\section{Detailed methods}

We now provide a detailed description of the methods used. We first describe the method used to simulate the spread of information and disease, which were used for both parameter selection and for producing the final results. We then detail the generation of random networks and the selection of parameters governing the transmission processes in these networks. Finally, we describe the modification of random networks into modular networks and comparisons of the effect of group size and modularity in these new networks and to the same processes in the random networks.

\section{Simulating disease and information spread}

We here summarise the methods used to model information and disease transmission throughout these simulations. We modelled both disease and information transmission as simple susceptible-infected (SI) models. Nodes were therefore either susceptible (uninfected/uninformed) or infected (informed), with no possibility for recovery from this state.

Disease-spread simulation We make the assumption that an individual's likelihood of infection depends on the infectiousness of the disease and how many of their contacts are infected. We begin by infecting a random node (i.e. individual) in the network. In each subsequent timestep, whether a susceptible node will become infected is determined by a binomial trial, with the probability of infection for a node $v$ being:

$1-(1-\beta)^{|N(v) \subseteq \mathrm{i}|}$

where $|N(v) \subseteq \mathrm{I}|$ is the number of nodes adjacent to node $v(N(v))$ that are infected. $\beta$ is a per timestep transmission probability per connection for a particular random network (see below for parameter selection information). The likelihood of a node becoming infected therefore increases as the number of infected connections increases (Fig. 2a).

Information-spread simulation We use a conformist learning rule to simulate the spread of information. Under this rule, an individual's likelihood of accepting the information will depend on a scaling parameter and the proportion of contacts who have already accepted the information, with the probability of accepting increasing once $50 \%$ of contacts have accepted the information. The likelihood of accepting the information based on the proportion of contacts who have accepted the information is therefore a sigmoidal function, described in the equation below and illustrated in Fig. 2b. Note that the function allows a very low probability of acquiring information even when no contacts are informed, which simulates there being some individual learning alongside social transmission of information. A random node starts with the information, and in each subsequent timestep whether a susceptible node $v$ will accept the information and become informed is based on a binomial trial, with the probability of accepting the information being

$\gamma \times \frac{1-2 * \eta}{1+\exp \left(-\psi \times \frac{1}{\gamma} \times\left(\frac{|N(v) \subseteq \mathrm{i}|}{\delta(v)}-0.5\right)\right)}+\eta$
Fig. 2 Visualisation of transmission probabilities for a) disease and b) information, depending on the number or proportion of infected/informed contacts, at different values of $R_{0}$ a)

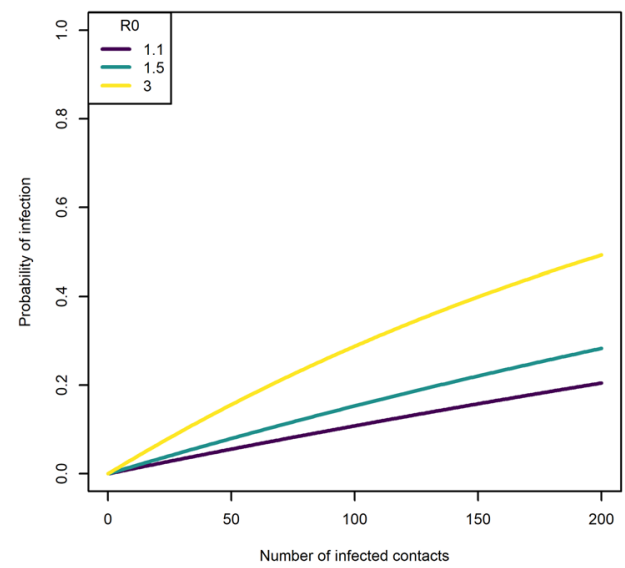

b)

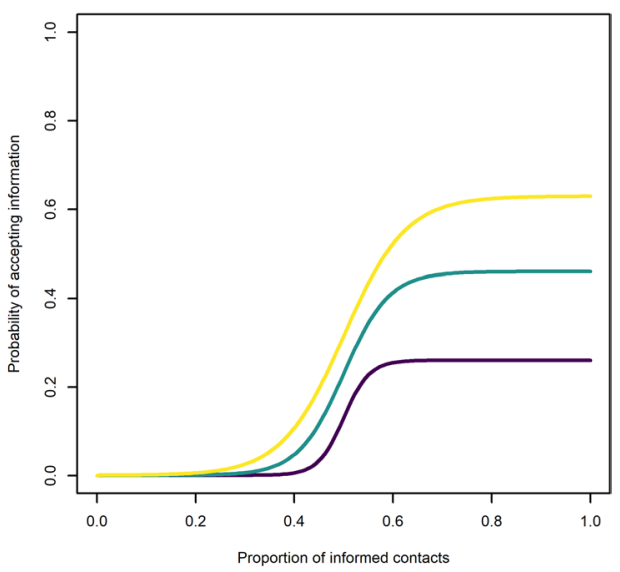


where $\gamma$ is a scaling parameter used to modify the rate of information spread (allowing us to ensure information and disease spread at the same speed in the random network), $|N(v) \subseteq i|$ is the number of adjacent nodes that are informed, and $\delta(v)$ is the node's degree. $\eta$ is the base probability of accepting information (baseline learning rate), and $\psi$ is the steepness of the sigmoidal function (Fig. 2b). Based on our exploration of parameter values to match the rate of spread of information to the rate of disease spread in the random networks, we fixed $\eta$ at 0.001 and $\psi$ at 10 in all simulations (Fig. 2).

\section{Random network generation and parameter selection}

We generated random networks consisting of 200 nodes using igraph's implementation of the Erdos-Renyi model (Csardi and Nepusz 2006). Probability of edge formation between nodes was determined by the value of the density parameter under investigation. Networks were unweighted (i.e. individuals were connected or not) and undirected (i.e. either both or neither individual were connected to each other). We then modelled the spread of a disease of each value of transmissibility (per-time period $R_{0}$; see Table 1 ) through this random network. This allowed us to establish a baseline disease spread speed in a random network of each density, which could later be compared to the spread speed in modular networks. We also used this spread of disease to choose parameters allowing information to spread at the same rate.

Disease spread parameter selection To simulate the spread of disease through a network, we converted a chosen pertime period $R_{0}$ to a transmission probability for the random network under investigation. First, we calculated $r$, the transmission probability for a time period $t$ in that network, where $t$ is the time period over which an $R_{0}$ is calculated and was fixed at 100 . For example, for an $R_{0}$ of 3 , an individual should on average infect 3 other individuals in this network over 100 timesteps.

$r=R_{0} / \frac{\left\langle k^{2}\right\rangle-\langle k\rangle}{\langle k\rangle}$

Here, $\langle k\rangle$ is the average degree of all nodes in the network and $\left\langle k^{2}\right\rangle$ is the average squared degree of all nodes in the network (Newman 2008). We then used the resulting value of $r$ to calculate $\beta$, the per timestep transmission probability per connection for this $R_{0}$ in this network.

$\beta=1-(1-r)^{(1 / t)}$

We used this value of $\beta$ when simulating disease spread through the random network under investigation and for all modular networks derived from that initial random network, for this value of our per-time period $R_{0}$ (Newman 2008).

Learning rule parameter selection Having established the value of $\beta$ that corresponded to a value of $R_{0}$ in the current random network, we then found the equivalent parameter value that lead to a similar transmissibility for information. To do this, we first simulated the spread of disease through the random network 50 times, recording the number of timesteps it took to infect $75 \%$ of the nodes. We used this distribution as a baseline with which to compare the spread of information. We searched for a value of a scaling parameter that governed the speed of information transmission, $\gamma$, which would produce a similar distribution of information spread (time to inform $75 \%$ of nodes) as our infection baseline. When generating a distribution, information spread through the random network was simulated 50 times with a potential value of $\gamma$, using the learning rule described below. We then compared the distribution of the number of timesteps to reach the $75 \%$ threshold from these 50 simulations of information spread to that of the 50 simulations of the spread of disease by calculating the Bhattacharyya distance (Bhattacharyya 1946) between the two distributions. Bhattacharyya distance is a measure of the similarity between two distributions using their means and variances. A smaller Bhattacharyya distance indicates a greater overlap between the two distributions. We optimised $\gamma$ so as to minimise the Bhattacharyya distance between the distribution of disease and information spread times to reach the 75\% threshold using R's "optimise" function (Nelder and Mead 1965). Parameter optimisation was run five times so as to avoid being trapped in local optima based on potential combinations of starting nodes and $\gamma$. From these five parameter searches, we selected the value of $\gamma$ that produced the smallest Bhattacharyya distance (i.e. resulted in information spreading at a similar speed to disease), and recorded the mean time taken for each to spread to $75 \%$ of individuals in the network.

\section{Modular network generation and comparisons}

We then altered the network to test how modularity and group sizes affected these spreading processes. For each random network, we generated 18 modular networks with differing levels of fragmentation, varying in group size and modularity. The random network was rewired to generate community structure with the desired group size and relative modularity $\left(Q_{\text {rel }}\right) \cdot Q_{\text {rel }}$ adjusts the modularity calculated for a particular network by dividing by the modularity calculated for the same network but within between-module edges excluded (Sah et al. 2017). The algorithm first assigns every node to a group, with the number of groups depending on the desired group size. It then rewires the network one edge 
at a time until the relative network modularity reaches its target. See Fig. 3 for an example of the modular networks generated for different densities and group sizes.

We then simulated information and disease spread through each of these 18 new modular networks 50 times, using the parameters $\beta$ and $\gamma$ derived from the random networks. We recorded the mean time it took for $75 \%$ of nodes to become infected and the mean time it took for $75 \%$ of nodes to become informed in each modular network, with a cut off if one or both of the spreading processes failed to reach this threshold after 3500 timesteps. Supplementary Fig. 2 shows a visualisation of the progress of the spreading processes through both random and modular networks, in relation to network density, and in the modular networks: modularity and group size. Supplementary Video 1 shows an animated example of simulations in both random and modular networks of varying densities. Supplementary Fig. 3 shows a static visualisation of the same set of simulations.

\section{Results}

\section{The impact of network modularity and fragmentation on transmission rate}

Speed of transmission of both disease and information was reduced by modular network structure, but mainly when group sizes were smaller and networks more fragmented (Fig. 4). The reduced speed of transmission was most striking in more modular networks ( $Q_{\text {rel }}$ of 0.6 and 0.8$)$. Importantly, more modular and fragmented networks slowed the transmission of infection far more than information for most network densities considered (Fig. 4; supplementary Fig. 4). Qualitatively similar patterns were apparent when considering the difference in spread speeds between modular and random networks (Fig. 5); both information and disease spread more slowly in modular networks than in random networks.

\section{The role of transmissibility and network density}

Higher levels of transmissibility always led to faster spread of disease and information, in all types of modular networks (Fig. 4). When transmissibility and modularity were high $\left(R_{0}=3\right.$ and $\left.Q_{\text {rel }} \geq 0.6\right)$, the time taken for $75 \%$ of the population to be informed remained effectively unchanged from that in random networks, while time taken for $75 \%$ to be infected increased substantially compared with the random networks except when network density was very low (Fig. 5). With lower modularity $\left(Q_{\text {rel }}\right.$ of 0.4$)$, only the smallest group sizes were able to slow transmission, and only when transmissibility was very low. There were 19 dense, highly modular networks with small group sizes where disease entirely failed to spread within 3500 timesteps when transmissibility was low ( $R_{0}$ of 1.1 or 1.25 ).

The time difference between $75 \%$ of the population becoming informed via conformist learning and $75 \%$ being infected increased with network density within the range

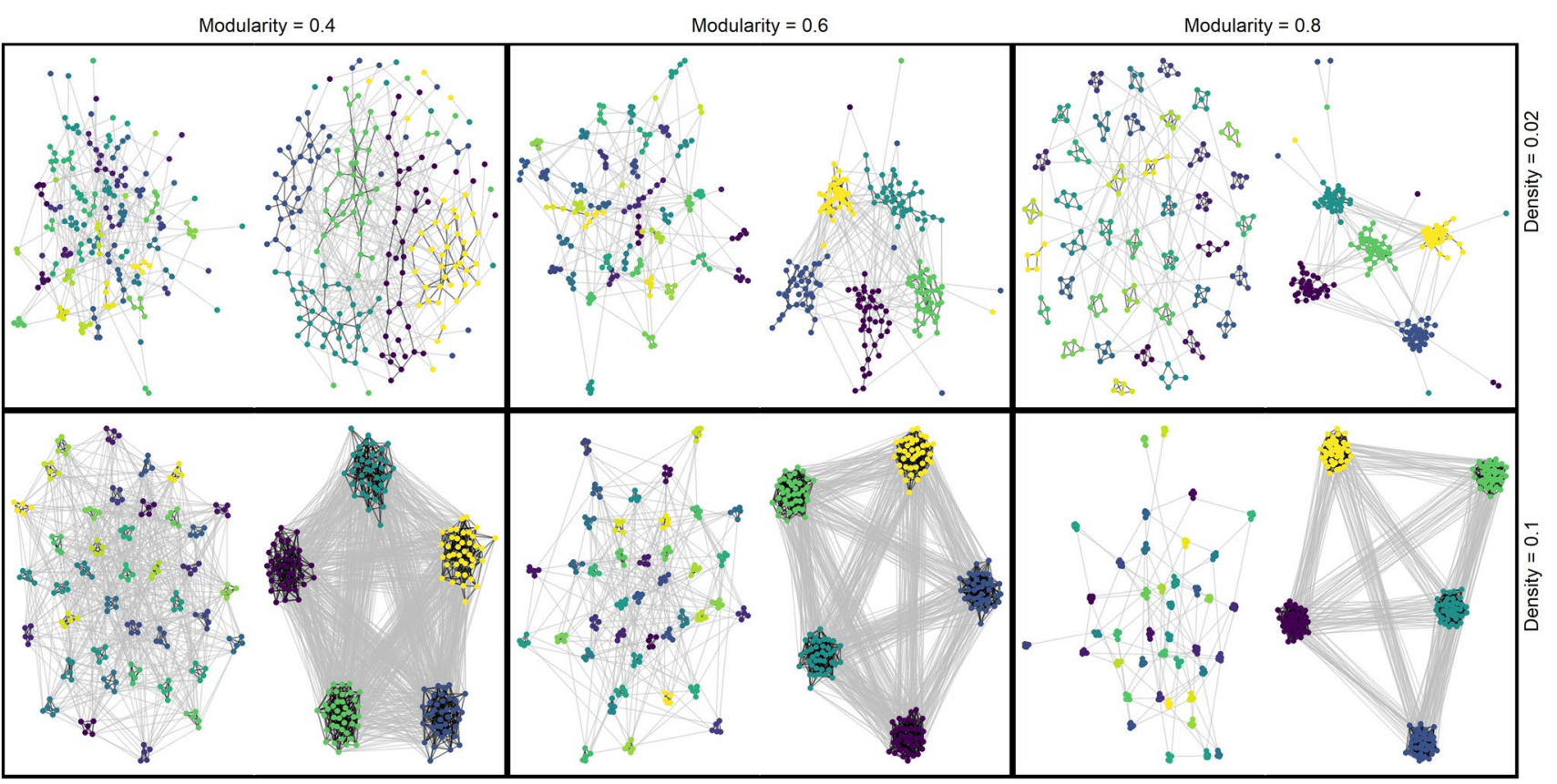

Fig. 3 Examples of the networks at different levels of network density (row), rewired to different values of network modularity (column) and two different group sizes (5 and 40) for the same population size (200) 

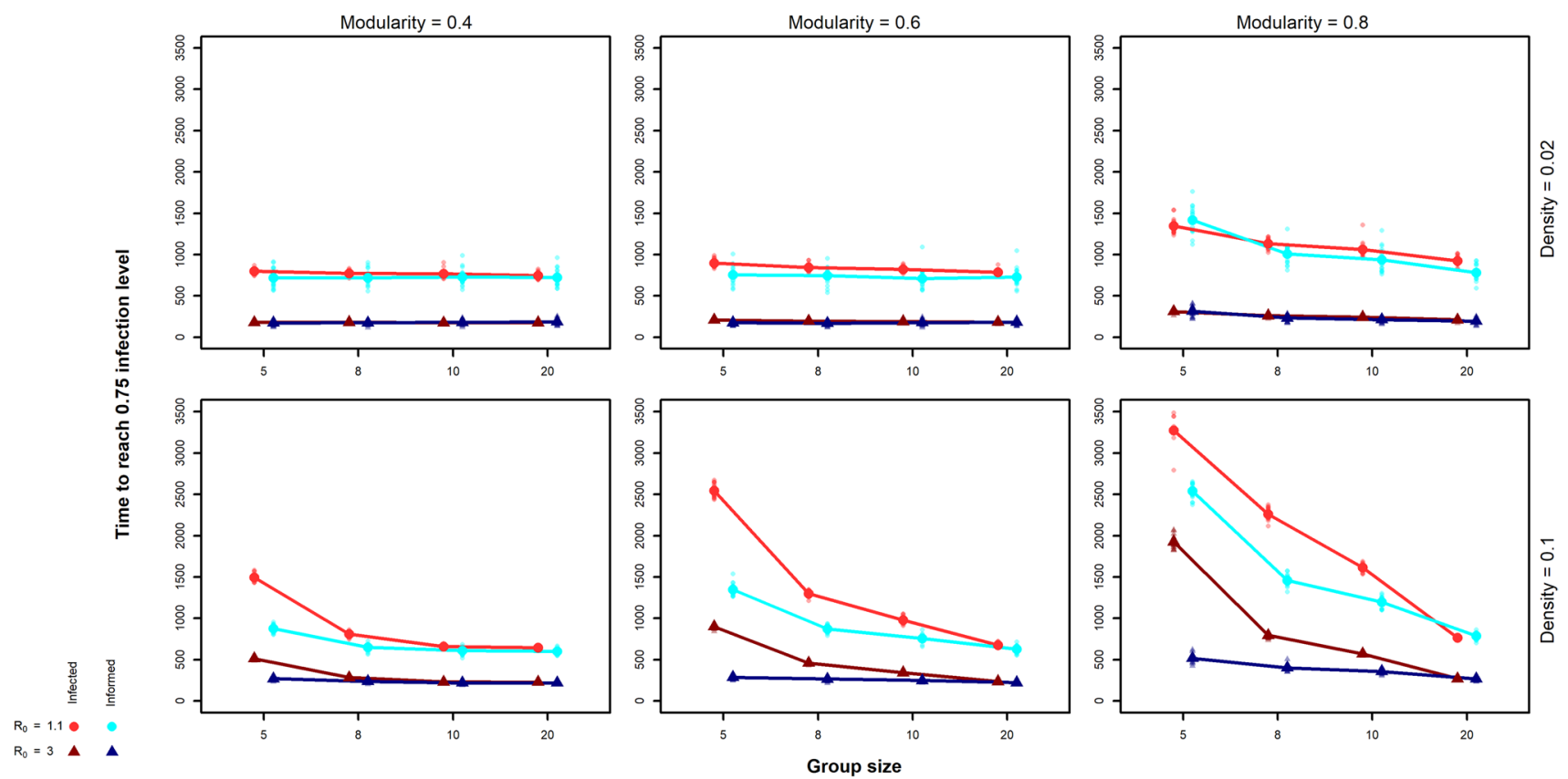

Fig. 4 Overview of mean time taken for disease (red) and information (blue) to infect $75 \%$ of nodes in modular networks of 200 nodes. Smaller datapoints are results for each of the 20 repeats, while large datapoints are the mean for that parameter combination. Results are shown for different levels of network modularity (column), density (row) and $R_{0}$ (symbol and colour). Note that for illustrative purposes,

studied (Fig. 6), although the nature of this relationship depended on both network modularity and transmissibility. When transmissibility was higher, the difference between the rate of information spread and rate of disease spread increased consistently as the modularity of the network increased (Fig. 6). How much the difference increased depended on the density of connections in the network. However, when transmissibility was lower, the relationship between modularity and difference in transmission speeds was more complicated. First, for networks with intermediate modularities ( $Q_{\text {rel }}$ of 0.4 or 0.6 ), the difference in the time taken for $75 \%$ of the population to be infected or informed by the two transmission processes showed a (slightly) humped relationship, with the difference in time taken being minimised (i.e. close to zero) at low- to intermediate network densities, with disease spreading faster than information in some repeats of the simulation. Second, at high network densities, the difference in transmission rates was higher for intermediate modularities $\left(Q_{\text {rel }}=0.6\right)$ than high modularities $\left(Q_{\text {rel }}=0.8\right)$, although it should be noted here that it was for networks with high densities, high modularities, and high fragmentation that disease failed to infect $75 \%$ of the population in some runs. Consequently, this result should be interpreted as a lack of evidence for a difference between intermediate and high modularities, rather than a quantitative difference between the two. we include only a subset of group sizes and other parameter values in this figure, while the full dataset is visualised in Supplementary Fig. 4. Note also that for infection spread, some runs of the simulation with a group size of five and network density of 0.1 did not complete before the time cut-off of our simulations and are not shown on this plot

For networks with intermediate or high modularity, the difference in the rate of transmission between information and infection was maximised for intermediate values of transmissibility (Fig. 7), resulting in the biggest difference at $R_{0}=1.5$ for networks with $Q_{\text {rel }}=0.6$ (intermediate modularity) and $R_{0}=2$ for networks with $Q_{\text {rel }}=0.8$ (high modularity) among the parameter values we tested.

\section{Discussion}

We show that both modularity and network fragmentation/ (sub-)group size are important in mediating the balance between pathogen and information spread when individuals use conformist social learning. In our simulations, it took longer for three quarters of individuals to become either informed or infected in more fragmented modular networks where group sizes were small, but not when group sizes were larger. In more fragmented modular networks, the rate of information transmission slowed less than that of infectious disease compared with random networks, resulting in information spreading faster than infection.

Our simulations clearly demonstrate that modularity can, but does not always, slow the rate of spread of infection, supporting the findings of previous work (Salathé and Jones 2010; Griffin and Nunn 2012; Nunn et al. 2015; Sah et al. 

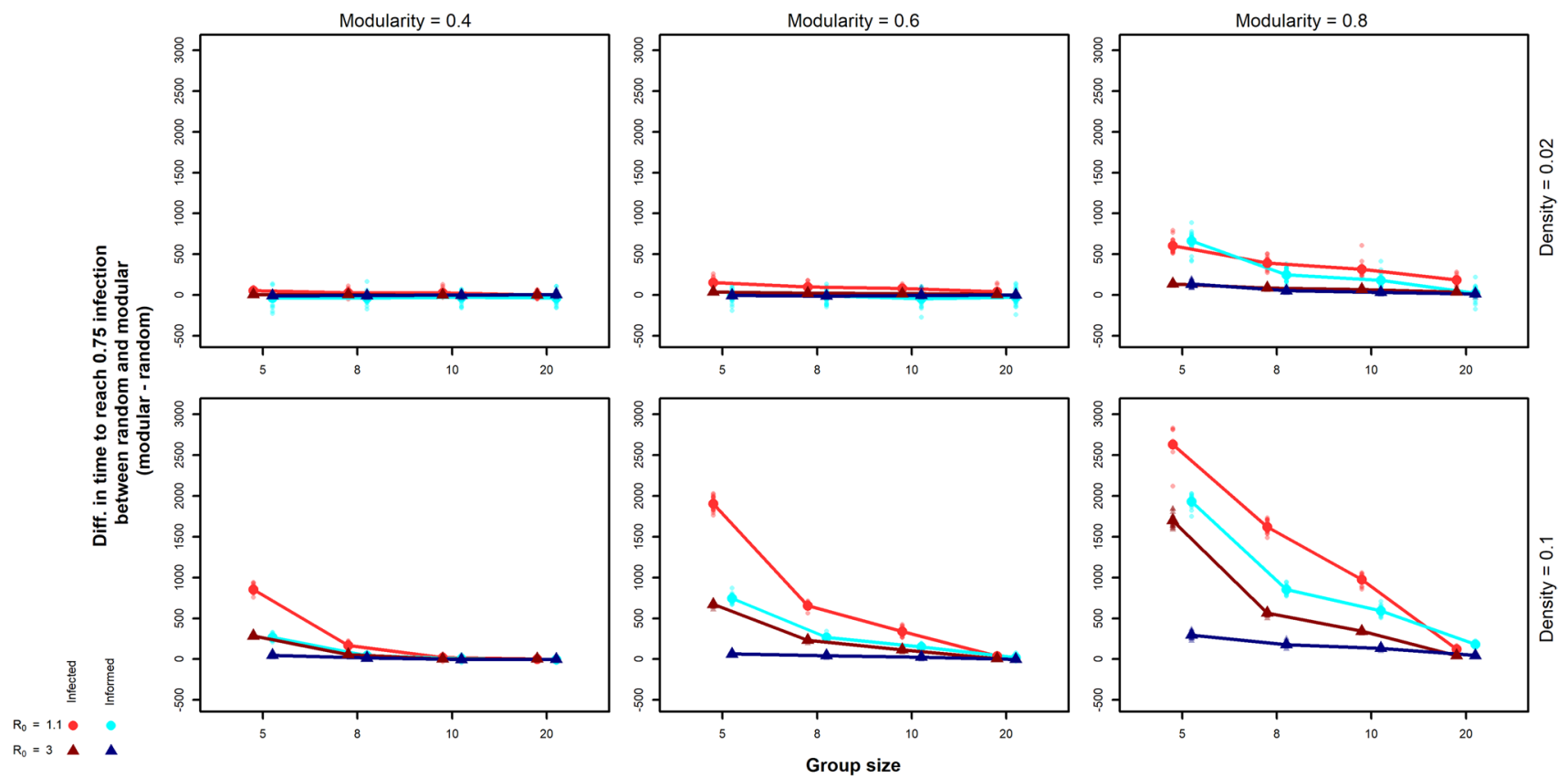

Fig. 5 Overview of the difference between modular and random networks in mean time taken for disease (red) and information (blue) to infect $75 \%$ of nodes in a network of 200 nodes. The $Y$-axis shows the mean time taken for a transmission process to reach $75 \%$ of nodes in a random network subtracted from the mean time taken for a transmission process to reach $75 \%$ of nodes in a modular network derived from that random network. Positive values therefore indicate slower spreading in modular networks, negative values faster spreading. Smaller datapoints are results from each of the 20 repeats, while large datapoints are the means for that parameter combination. Results are shown for different levels of network modularity (column), density (row), and $R_{0}$ (symbol and colour). Note that we include only a subset of group sizes and other parameter values in this figure; for a figure showing the full dataset, see supplementary Fig. 5. Note also that for infection spread, some runs of the simulation with a group size of five and network density of 0.1 did not complete before the time cut-off of our simulations and are not shown on this plot

et al. 2014; Romano et al. 2018). In our results, there was no consistent tendency for information to spread faster in modular networks than in random networks. However, at low network densities and intermediate modularities $\left(Q_{\mathrm{rel}}\right.$ of 0.4 or 0.6 ), some individual simulation runs found faster information spread in modular than random networks. It is possible, therefore, that modularity might facilitate faster information spread in our modelling framework when using parameter values that we did not explore in this study, such as values of $\psi$ that result in a steeper threshold for conformist learning (González-Avella et al. 2011; Nematzadeh et al. 2014). Previously, network efficiency (a proxy for how efficiently a network exchanges information) was shown to have a relatively weak relationship with modularity that peaked at smaller levels of modularity than those investigated here (Romano et al. 2018). Together with our findings, this suggests that information spreads fastest in these less modular networks, while more modular networks favour the rate of information transmission relative to the spread of infection.

Conformist social learning has been reported in species ranging from vervet monkeys to great tits and fruit flies (van de Waal et al. 2013; Aplin et al. 2015; Danchin et al. 2018), suggesting that the difference between information 


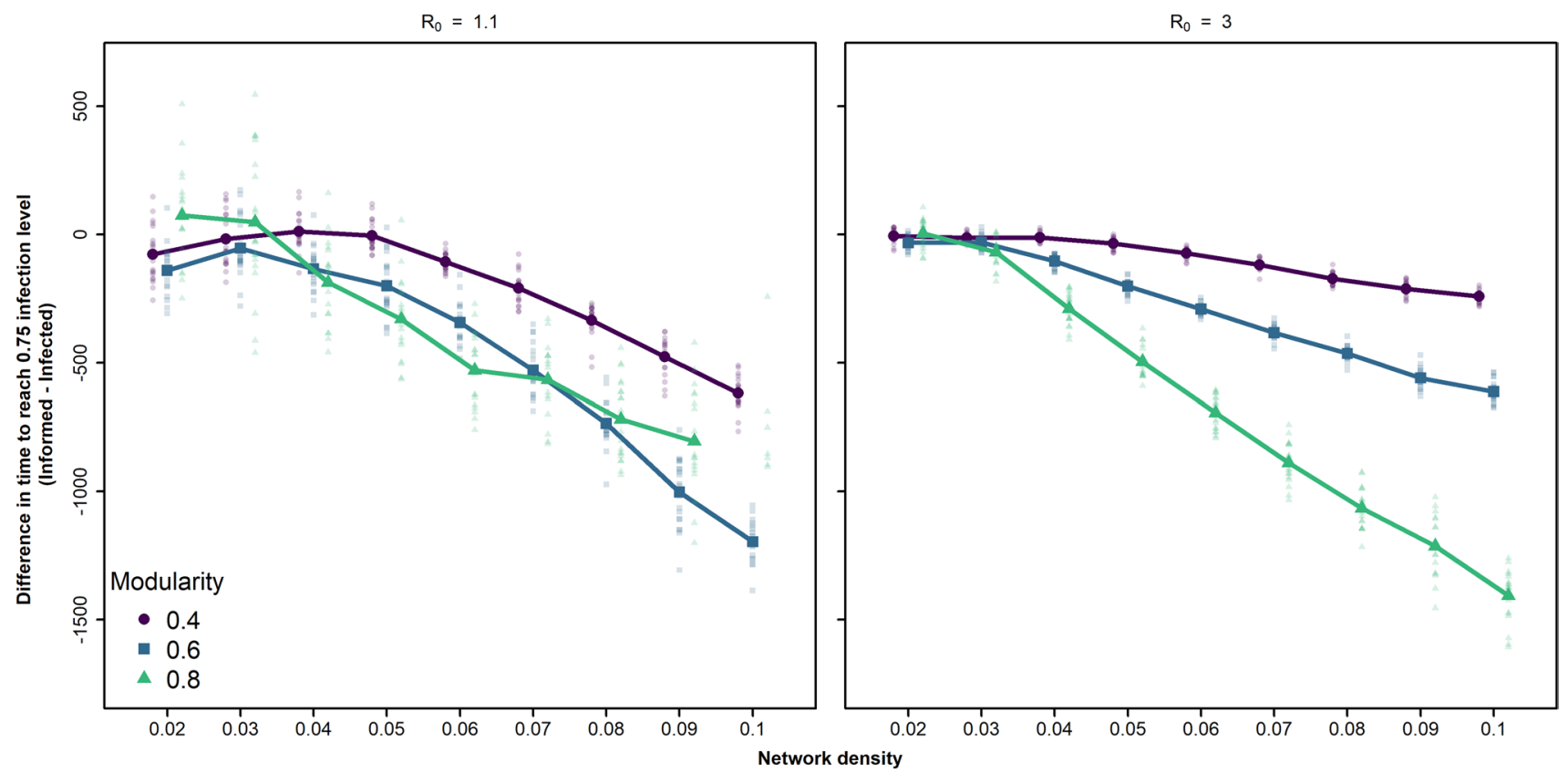

Fig. 6 Effect of network density on the difference in time taken for disease and information to infect $75 \%$ of 200 nodes in a modular network consisting of small groups of 5 individuals. The $Y$-axis shows the time taken for disease to infect $75 \%$ of nodes subtracted from the time taken for information to inform $75 \%$ of nodes. Positive values on the $Y$-axis therefore indicate information taking longer to reach this

transmission through conformist learning and infectious disease spread shown in our models has ecological and evolutionary significance. Social groups with modular social structure and sufficiently small sub-groups will impede the transmission of infection while having much less impact on how quickly beneficial information spreads. The importance of modular social structure will be most apparent when infection and information spread through similar or overlapping types of interaction, for example when social learning requires prolonged close proximity interactions (Hobaiter et al. 2014). The fact that the difference in information and disease spread is substantial only for small (sub-)groups may contribute to the evolution of hierarchical or multi-level social structures as seen in humpback whales and vulturine guineafowl (Cantor et al. 2015; Papageorgiou et al. 2019; Grueter et al. 2020). In addition, our work suggests capacity for social structure and social learning rules to co-evolve We might expect conformist social learning to be more common in species with hierarchical social structures, in which the social networks of stable groups are further subdivided by fission-fusion dynamics (e.g. chimpanzees Pan troglodytes and Pan paniscus: Lehmann et al. 2007; spider-monkeys Ateles geoffroyi: Ramos-Fernández and Morales 2014). Such social structures could lead to highly fragmented networks which increase the effectiveness of conformist learning relative to simple contagions. Finally, our findings will also be level of infection than disease, while negative values on the $Y$-axis indicate disease taking longer. Smaller datapoints are raw data, while large datapoints are means. Results are shown for different levels of $R_{0}$, network modularity and density. Means for parameter combinations where $75 \%$ of individuals were not infected by our cut-off of 3500 time steps are excluded

relevant to the spread of information about a disease (Funk et al. 2009). When sub-groups and small networks have intermediate modularity, information about the presence of disease will be able to spread faster than the infection itself, helping to enhance behavioural or social immunity (De Roode and Lefèvre 2012; Meunier 2015). The difference will be greatest when the density of connections in a group is higher, as might be expected, for example, in a social insect colony or when comparing smaller human communities (higher network fragmentation) to large cities (networks likely still modular but with much larger groups). Future research that models direct interactions between information and disease spreading simultaneously (Funk et al. 2009; Silk et al. 2021) could also contribute to understanding patterns of social behaviour in animal groups.

Our results also complement recent modelling and empirical research suggesting modular networks have minimal effect on the spread of social information (Cantor et al. 2021; Laker et al. 2021). For example, Laker et al. (2021) showed that the transmission of novel foraging information was not impacted by the modularity of network structure in domestic fowl chicks Gallus gallus domesticus. If we assume conformist learning, our models show that for the modularities investigated in their study $(Q=0.63-0.73)$, we would predict either no increase or a relatively small increase in the rate of learning, depending on how easy information is to 


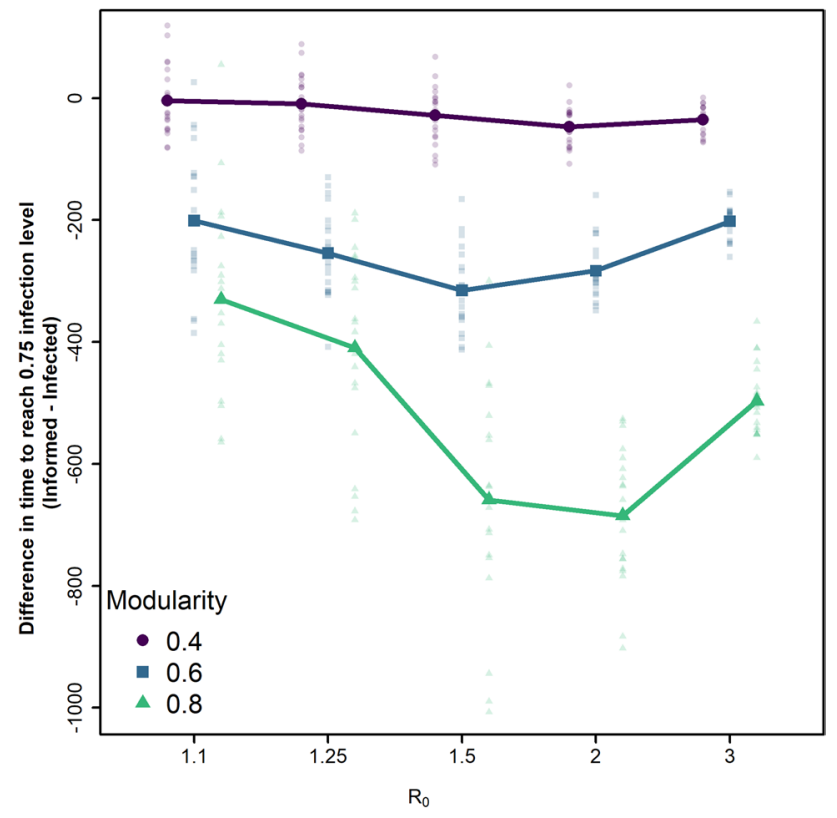

Fig. 7 Effect of transmissibility on the difference in time taken for disease and information to infect $75 \%$ of nodes in a 200 -node network of density 0.05 with small groups of 5 individuals. The $Y$-axis shows the time taken for disease to infect $75 \%$ of nodes subtracted from the time taken for information to inform $75 \%$ of nodes. Positive values on the $Y$-axis therefore indicate information taking longer to reach this level of infection than disease, while negative values on the $Y$-axis indicate disease taking longer. Smaller datapoints are raw data, while large datapoints are means. Results are shown for different levels of network modularity

acquire; the easier and faster it is to acquire information, the less impact even small sub-groupings have on how quickly information spreads. However, information that spreads according to other social learning rules may respond differently to more modular social structures.

There are a number of limitations to our current model that would benefit from further development. We have only considered binary, unweighted networks at each time-step (a social connection either exists or not). In many situations, social connections will vary in strength between different individuals, which could influence the likelihood of transmission (Valsecchi et al. 1996; Swaney et al. 2001). Similarly, we have only considered one type of social learning rule, while in reality, individuals may use other learning rules such as simple learning rules that result in information spreading in a way very similar to disease (Kempe et al. 2003; van Schaik 2010), a bias toward learning from kin or familiar individuals (e.g. deer mice Peromyscus maniculatus: Kavaliers et al. 2005; chimpanzees Pan troglodytes: Kendal et al. 2015; zebra finches Taeniopygia guttata: Boogert et al. 2018), or combinations of multiple rules. A more comprehensive exploration that incorporates heterogeneity in how individuals learn would provide additional insight into how information spread might be influenced by group size and fragmentation. Our model also uses a static network, with no changes to edge structure over time. In reality, it is likely that network dynamics will influence how information and infection spread through a population, with both transmission types potentially causing changes in network structure (Funk et al. 2009; Croft et al. 2011; Kulahci et al. 2018; Stockmaier et al. 2020). For example, the position of individuals within a network might be expected to change when they are infected (Lopes et al. 2016; Stockmaier et al. 2020) or become informed (Kulahci et al. 2018). Future models that incorporate these network dynamics are likely to provide further insights into the patterns demonstrated here. To aid comparison of the effect of network structure on the two transmission processes, we also set the spread speeds of the two processes to be as similar as possible. Equivalent transmissibility is unlikely in the real world, and so empirical studies are necessary to help explore when and how our simulation results apply to real networks. Preferably, these studies would use systems where interactions can be observed and network structure experimentally manipulated (Blonder and Dornhaus 2011; Stroeymeyt et al. 2018).

Our simulations also focussed solely on a susceptibleinfected model where individuals remained infected or informed for the rest of the simulation. This approximated the early stages of spread through a group, helping us directly compare the spread of infection and information. However, modelling recovery from infection using a susceptible-infected-recovered (SIR) model (e.g. with a fixed infectious period for the disease) or allowing individuals to return to a susceptible or uninformed state (using a susceptibleinfected-susceptible [SIS] model) would also be informative. Modularity would be expected to have a bigger impact on infectious disease spread if recovery was included, as outbreaks may become trapped within particular modules (Sah et al. 2017). In an SIS version, the ease with which individuals in a (sub-)group become re-informed may depend on the synchrony with which they became uninformed; if a single individual became uninformed it would be likely to re-learn the information very rapidly with conformist learning; however, if a whole (sub-)group became uninformed simultaneously, then we might expect it to take much longer for them to re-learn the information.

Overall, by demonstrating the relative speed with which infection and information can saturate animal groups across a range of biologically realistic social structures, our research helps us learn more about how infection and information act together as selection pressures in animal societies. Groups with social structures that favour the spread of information over infection are more likely to persist, and so the social behaviour of their members be selected for, potentially playing a key role in the evolution of various 
social systems. Our results highlight the importance of considering network fragmentation in addition to modularity when studying the ecological consequences of animal social network structure and their implications for the evolution of sociality.

Supplementary Information The online version contains supplementary material available at https://doi.org/10.1007/s00265-021-03102-4.

Acknowledgements We would like to thank Coby Thompson-Knight for their contribution to preliminary work related to this study. We would also like to thank Barbara König, Jordi Bascompte, and Nina Fefferman for their support during the writing of the paper. We thank anonymous reviewers for their useful comments and suggestions.

Author contribution All authors conceived the study. JCE and MJS coded and ran the simulations and wrote the manuscript. All authors discussed the results and contributed to the final manuscript.

Funding JCE is funded by a University of Zurich Forschungskredit grant. NJB is funded by a Royal Society Dorothy Hodgkin Research Fellowship (DH140080). DJH is funded by the University of Exeter.

Data availability Simulation results are available in the supplementary materials.

Code availability Simulation code is available in the supplementary materials.

\section{Declarations}

Conflict of interest The authors declare no competing interests.

\section{References}

Adelman JS, Moyers SC, Farine DR, Hawley DM (2015) Feeder use predicts both acquisition and transmission of a contagious pathogen in a North American songbird. Proc R Soc B 282:20151429

Allen J, Weinrich M, Hoppitt W, Rendell L (2013) Network-based diffusion analysis reveals cultural transmission of lobtail feeding in humpback whales. Science 340:485-488

Aplin LM, Farine DR, Morand-Ferron J, Cockburn A, Thornton A, Sheldon BC (2015) Experimentally induced innovations lead to persistent culture via conformity in wild birds. Nature 518:538-541

Aplin LM, Farine DR, Morand-Ferron J, Sheldon BC (2012) Social networks predict patch discovery in a wild population of songbirds. Proc R Soc Lond B 279:4199-4205

Beauchamp G, Alexander P, Jovani R (2012) Consistent waves of collective vigilance in groups using public information about predation risk. Behav Ecol 23:368-374

Berdahl AM, Kao AB, Flack A, Westley PAH, Codling EA, Couzin ID, Dell AI, Biro D (2018) Collective animal navigation and migratory culture: from theoretical models to empirical evidence. Phil Trans R Soc B 373:20170009

Bhattacharyya A (1946) On a measure of divergence between two multinomial populations. Sankhyā 7:401-406

Blonder B, Dornhaus A (2011) Time-ordered networks reveal limitations to information flow in ant colonies. PLoS ONE 6:e20298
Boogert NJ, Lachlan RF, Spencer KA, Templeton CN, Farine DR (2018) Stress hormones, social associations and song learning in zebra finches. Phil Trans R Soc B 373:20170290

Cantor M, Chimento M, Smeele SQ, He P, Papageorgiou D, Aplin LM, Farine DR (2021) Social network architecture and the tempo of cumulative cultural evolution. Proc R Soc B 288:20203107

Cantor M, Shoemaker LG, Cabral RB, Flores CO, Varga M, Whitehead H (2015) Multilevel animal societies can emerge from cultural transmission. Nat Commun 6:8091

Centola D (2010) The spread of behavior in an online social network experiment. Science 329:1194-1197

Clutton-Brock T (2016) Mammal societies. John Wiley \& Sons, Chichester

Côté IM, Poulinb R (1995) Parasitism and group size in social animals: a meta-analysis. Behav Ecol 6:159-165

Couzin ID, Laidre ME (2009) Fission-fusion populations. Curr Biol 19:R633-R635

Crane AL, Ferrari MCO (2013) Social learning of predation risk: a review and prospectus. In: Clark $\mathrm{K}$ (ed) Social learning theory: phylogenetic considerations across animal, plant, and microbial taxa. Nova Science Publishers, New York, pp 53-82

Croft DP, Edenbrow M, Darden SK, Ramnarine IW, van Oosterhout C, Cable J (2011) Effect of gyrodactylid ectoparasites on host behaviour and social network structure in guppies Poecilia reticulata. Behav Ecol Sociobiol 65:2219-2227

Csardi G, Nepusz T (2006) The igraph software package for complex network research. Int J Complex Syst 1695:1-9

Danchin E, Giraldeau L-A, Valone TJ, Wagner RH (2004) Public information: from nosy neighbors to cultural evolution. Science 305:487-491

Danchin E, Nöbel S, Pocheville A et al (2018) Cultural flies: conformist social learning in fruitflies predicts long-lasting mate-choice traditions. Science 362:1025-1030

Daszak P, Cunningham AA, Hyatt AD (2000) Emerging infectious diseases of wildlife - threats to biodiversity and human health. Science 287:443-449

Davis S, Abbasi B, Shah S, Telfer S, Begon M (2015) Spatial analyses of wildlife contact networks. Interface 12:20141004

JC Roode De T Lefèvre 2012 Behavioral Immunity in Insects Insects 3789820

Drewe J, Madden J, Pearce G (2009) The social network structure of a wild meerkat population: 1. Inter-Group Interactions Behav Ecol Sociobiol 63:1295-1306

Evans JC, Morand-Ferron J (2019) The importance of preferential associations and group cohesion: constraint or optimality. Behav Ecol Sociobiol 73:109

Evans JC, Silk MJ, Boogert NJ, Hodgson DJ (2020) Infected or informed? Social structure and the simultaneous transmission of information and infectious disease. Oikos 129:1271-1288

Firth JA (2020) Considering complexity: animal social networks and behavioural contagions. Trends Ecol Evol 35:100-104

Frank SA (2007) All of life is social. Curr Biol 17:R648-R650

Frechette JL, Sieving KE, Boinski S (2014) Social and personal information use by squirrel monkeys in assessing predation risk. Am J Primatol 76:956-966

Funk S, Gilad E, Watkins C, Jansen VA (2009) The spread of awareness and its impact on epidemic outbreaks. P Natl Acad Sci USA 106:6872-6877

Godfrey SS (2013) Networks and the ecology of parasite transmission: a framework for wildlife parasitology. Int J Parasitol 2:235-245

Godfrey SS, Bull CM, James R, Murray K (2009) Network structure and parasite transmission in a group living lizard, the gidgee skink, Egernia stokesii. Behav Ecol Sociobiol 63:1045-1056

González-Avella JC, Eguíluz VM, Marsili M, Vega-Redondo F, San Miguel M (2011) Threshold learning dynamics in social networks. PLOS ONE 6:e20207 
Gosling LM, Roberts SC (2001) Scent-marking by male mammals: cheat-proof signals to competitors and mates. Adv Stud Behav 30:169-217

Griffin RH, Nunn CL (2012) Community structure and the spread of infectious disease in primate social networks. Evol Ecol 26:779-800

Grueter CC, Qi X, Zinner D, Bergman T, Li M, Xiang Z, Zhu P, Migliano AB, Miller A, Krützen M (2020) Multilevel organisation of animal sociality. Trends Ecol Evol 35:834-847

Hobaiter C, Poisot T, Zuberbühler K, Hoppitt W, Gruber T (2014) Social network analysis shows direct evidence for social transmission of tool use in wild chimpanzees. PLoS Biol 12:e1001960

Hoyt JR, Langwig KE, White JP et al (2018) Cryptic connections illuminate pathogen transmission within community networks. Nature 563:710-713

Ilany A, Akcay E (2016) Social inheritance can explain the structure of animal social networks. Nat Commun 7:12084

Kavaliers M, Colwell DD, Choleris E (2005) Kinship, familiarity and social status modulate social learning about "micropredators" (biting flies) in deer mice. Behav Ecol Sociobiol 58:60-71

Kempe D, Kleinberg J, Tardos É (2003) Maximizing the spread of influence through a social network. In: Proceedings of the ninth ACM SIGKDD international conference on Knowledge discovery and data mining. ACM, New York, pp 137-146

Kendal R, Hopper LM, Whiten A, Brosnan SF, Lambeth SP, Schapiro SJ, Hoppitt W (2015) Chimpanzees copy dominant and knowledgeable individuals: implications for cultural diversity. Evol Hum Behav 36:65-72

Kendal RL, Boogert NJ, Rendell L, Laland KN, Webster M, Jones PL (2018) Social learning strategies: bridge-building between fields. Trends Cogn Sci 22:651-665

Kerth G, König B (1999) Fission, fusion and nonrandom associations in female Bechstein's bats (Myotis bechsteinii). Behaviour 136:1187-1202

Kramer J, Meunier J (2019) The other facets of family life and their role in the evolution of animal sociality. Biol Rev 94:199-215

Krause J, Croft DP, James R (2007) Social network theory in the behavioural sciences: potential applications. Behav Ecol Sociobiol 62:15-27

Krause J, Ruxton GD, Ruxton GD, Ruxton IG (2002) Living in groups. Oxford University Press, Oxford

Kulahci IG, Ghazanfar AA, Rubenstein DI (2018) Knowledgeable lemurs become more central in social networks. Curr Biol 28:1306-1310

Laker PR, Hoppitt W, Weiss M, Madden JR (2021) The modularity of a social group does not affect the transmission speed of a novel, socially learned behaviour, or the formation of local variants. Proc R Soc B 288:20202614

Laland KN (2004) Social learning strategies. Learn Behav 32:4-14

Lehmann J, Korstjens AH, Dunbar RIM (2007) Fission-fusion social systems as a strategy for coping with ecological constraints: a primate case. Evol Ecol 21:613-634

Lloyd-Smith JO, Schreiber SJ, Kopp PE, Getz WM (2005) Superspreading and the effect of individual variation on disease emergence. Nature 438:355-359

Lopes PC, Block P, König B (2016) Infection-induced behavioural changes reduce connectivity and the potential for disease spread in wild mice contact networks. Sci Rep 6:31790

Macy MW (1991) Chains of cooperation: threshold effects in collective action. Am Sociol Rev 56:730-747

McGregor PK (2005) Animal communication networks. Cambridge University Press, Cambridge

Meunier J (2015) Social immunity and the evolution of group living in insects. Phil Trans R Soc B 370:20140102

Moore C, Newman ME (2000) Epidemics and percolation in smallworld networks. Phys Rev E 61:5678
Nelder JA, Mead R (1965) A simplex method for function minimization. Comput J 7:308-313

Nematzadeh A, Ferrara E, Flammini A, Ahn Y-Y (2014) Optimal network modularity for information diffusion. Phys Rev Lett 113:088701

Newman ME (2008) The mathematics of networks. In: Durlauf SN, Blume LE (eds) The New Palgrave Encyclopedia of Economics, 2nd edn. Mcmillan, New York, pp 1-12

Nöbel S, Allain M, Isabel G, Danchin E (2018) Mate copying in Drosophila melanogaster males. Anim Behav 141:9-15

Nunn CL, Jordán F, McCabe CM, Verdolin JL, Fewell JH (2015) Infectious disease and group size: more than just a numbers game. Phil Trans R Soc B 370:20140111

Papageorgiou D, Christensen C, Gall GE, Klarevas-Irby JA, Nyaguthii B, Couzin ID, Farine DR (2019) The multilevel society of a small-brained bird. Curr Biol 29:R1120-R1121

Pinter-Wollman N, Hobson EA, Smith JE, Edelman AJ, Shizuka D, De Silva S, Waters JS, Prager SD, Sasaki T, Wittemyer G (2014) The dynamics of animal social networks: analytical, conceptual, and theoretical advances. Behav Ecol 25:242-255

R Development Core Team (2020) R: A Language and Environment for Statistical Computing. R Foundation for Statistical Computing, Vienna, Austria, http://www.R-project.org

Ramos-Fernández G, Morales JM (2014) Unraveling fissionfusion dynamics: how subgroup properties and dyadic interactions influence individual decisions. Behav Ecol Sociobiol 68:1225-1235

Romano V, MacIntosh AJJ, Sueur C (2020) Stemming the flow: information, infection, and social evolution. Trends Ecol Evol 35:849-853

Romano V, Shen M, Pansanel J, MacIntosh AJ, Sueur C (2018) Social transmission in networks: global efficiency peaks with intermediate levels of modularity. Behav Ecol Sociobiol 72:154

Rozins C, Silk MJ, Croft DP, Delahay RJ, Hodgson DJ, McDonald RA, Weber N, Boots M (2018) Social structure contains epidemics and regulates individual roles in disease transmission in a group-living mammal. Ecol Evol 8:12044-12055

Sah P, Leu ST, Cross PC, Hudson PJ, Bansal S (2017) Unraveling the disease consequences and mechanisms of modular structure in animal social networks. P Natl Acad Sci USA 114:4165-4170

Salathé M, Jones JH (2010) Dynamics and control of diseases in networks with community structure. PLoS Comput Biol 6:e1000736

Silk MJ, Carrignon S, Bentley RA, Fefferman NH (2021) Improving pandemic mitigation policies across communities through coupled dynamics of risk perception and infection. Proc R Soc B 288:20210834

Silk MJ, Croft DP, Delahay RJ, Hodgson DJ, Weber N, Boots M, McDonald RA (2017) The application of statistical network models in disease research. Methods Ecol Evol 8:1026-1041

Silk MJ, Croft DP, Tregenza T, Bearhop S (2014) The importance of fission-fusion social group dynamics in birds. Ibis 156:701-715

Spiegel O, Leu ST, Sih A, Bull CM (2016) Socially interacting or indifferent neighbours? Randomization of movement paths to tease apart social preference and spatial constraints. Methods Ecol Evol 7:971-979

Stattner E, Vidot N (2011) Social network analysis in epidemiology: current trends and perspectives. In: 2011 Fifth International Conference on Research Challenges in Information Science. IEEE, pp 1-11

Stockmaier S, Bolnick DI, Page RA, Carter GG (2020) Sickness effects on social interactions depend on the type of behaviour and relationship. J Anim Ecol 89:1387-1394

Stroeymeyt N, Grasse AV, Crespi A, Mersch DP, Cremer S, Keller L (2018) Social network plasticity decreases disease transmission in a eusocial insect. Science 362:941-945 
Swaney W, Kendal J, Capon H, Brown C, Laland KN (2001) Familiarity facilitates social learning of foraging behaviour in the guppy. Anim Behav 62:591-598

Valsecchi P, Choleris E, Moles A, Guo C, Mainardi M (1996) Kinship and familiarity as factors affecting social transfer of food preferences in adult Mongolian gerbils (Meriones unguiculatus). J Comp Psychol 110:243

van de Waal E, Borgeaud C, Whiten A (2013) Potent social learning and conformity shape a wild primate's foraging decisions. Science 340:483-485

van de Waal E, Renevey N, Favre CM, Bshary R (2010) Selective attention to philopatric models causes directed social learning in wild vervet monkeys. Proc R Soc Lond B 277:2105-2111

van Schaik CP (2010) Social learning and culture in animals. In: Kappeler P (ed) Animal behaviour: evolution and mechanisms. Springer, Berlin, pp 623-653

VanderWaal KL, Wang H, McCowan B, Fushing H, Isbell LA (2014) Multilevel social organization and space use in reticulated giraffe (Giraffa camelopardalis). Behav Ecol 25:17-26
Weber N, Carter SP, Dall SRX, Delahay RJ, McDonald JL, Bearhop S, McDonald RA (2013) Badger social networks correlate with tuberculosis infection. Curr Biol 23:R915-R916

Webster MM, Atton N, Hoppitt WJE, Laland KN (2013) Environmental complexity influences association network structure and network-based diffusion of foraging information in fish shoals. Am Nat 181:235-244

Wey T, Blumstein DT, Shen W, Jordán F (2008) Social network analysis of animal behaviour: a promising tool for the study of sociality. Anim Behav 75:333-344

White LA, Forester JD, Craft ME (2017) Using contact networks to explore mechanisms of parasite transmission in wildlife. Biol Rev 92:389-409

Publisher's note Springer Nature remains neutral with regard to jurisdictional claims in published maps and institutional affiliations. 